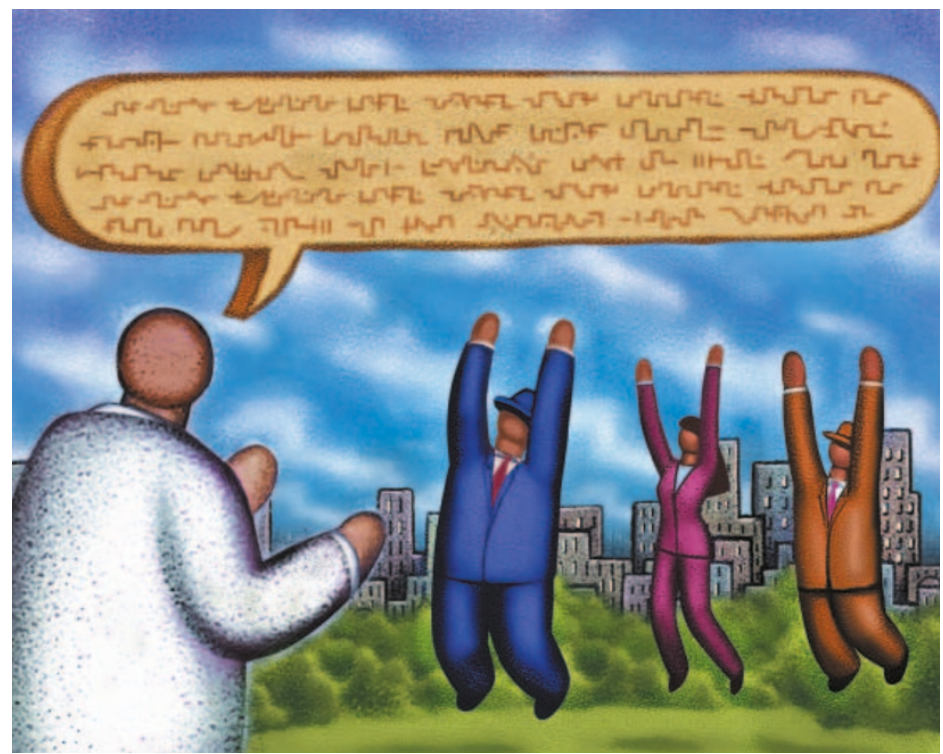

T-CELL SIGNALLING

\section{Giving T cells a PEP talk}

A recent report in Science shows that PEST-domain-enriched tyrosine phosphatase (PEP) has a specific role in dampening the response of effector/memory $\mathrm{T}$ cells to T-cell receptor (TCR) stimulation. Like a teacher curbing the activity of a boisterous child, PEP can calm the response of experienced $\mathrm{T}$ cells to antigenic stimulation.

Pep-deficient mice had no obvious changes in lymphoid organs in early age, but developed splenomegaly and lymphadenopathy at ages greater than 6 months. This was due to an increased number of both $\mathrm{CD}^{+}$and $\mathrm{CD}^{+}$effector and memory $\mathrm{T}$ cells. The initial activation of wild-type and Pep-deficient naive T cells was similar; but 3-4 days after TCR stimulation, the Pep-deficient $T$ cells were cycling faster with no apparent change in the rate of activation-induced cell death, giving them a growth advantage over wild-type T cells.

Pep is known to inhibit the TCR signal cascade by dephosphorylating a crucial regulatory tyrosine residue on the protein tyrosine kinase Lck. The authors showed in vitro that wild-type effector $\mathrm{T}$ cells restimulated through the TCR showed transient phosphorylation of this Lck tyrosine, whereas Pep-deficient T cells had sustained phosphorylation. For the Pep-deficient $\mathrm{T}$ cells, this resulted in prolonged and increased activation of downstream mitogen-activated protein kinases. When wild-type or Pep-deficient naive
TCR-transgenic T cells were transferred to irradiated mice and then stimulated with specific antigen, the Pep-deficient T-cell populations expanded much faster, confirming that Pep has a similar inhibitory effect in vivo. The wild-type and contracted at a similar rate after antigen challenge, again showing that Pep has no effect on cell death.

The increased number of effector/memory T cells in Pep-deficient mice was associated with the development of germinal centres in the spleen and Peyer's patches. This B-cell effect was shown to be partly a result of increased T-cell cooperation, although further studies of Pep in B cells are required. However, none of the mice autoimmune disease, leading Chan and colleagues to suggest that lack of function of Pep could only act as an enhancer for autoimmunity by increasing T-cell clonal expansion after a primary initiating defect that affected self-tolerance. Pep might be one of the many checkpoints that our immune systems have developed to prevent an inappropriate, possibly self-reactive, response. By contrast, blocking the activity of Pep might enhance the response to vaccination.

Kirsty Minton

(2) References and links ORIGINAL RESEARCH PAPER Hasegawa, K. et al. PEST domain-enriched tyrosine phosphatase (PEP) regulation of effector/memory T cells. Pep-deficient T-cell populations had any evidence of autoantibodies or Science 303, 685-689 (2004)
H I G H L I G H T S

\section{IN BRIEF}

\section{TOLERANCE}

AIRE functions as an E3 ubiquitin ligase.

Uchida, D. et al. J. Exp. Med. 199, 167-172 (2004)

Autoimmune regulator (AIRE) has an important role in the generation of self-tolerance, as mutations of this gene result in a range of organ-specific autoimmune diseases. Previous studies have indicated that AIRE is a transcriptional activator for the expression of otherwise organ-specific antigens by medullary epithelial cells of the thymus. This study now indicates that AIRE might also function as an E3 ubiquitin ligase. AIRE contains two plant homeodomains (PHDs), which have recently been shown in other proteins to facilitate polyubiquitylation of target proteins. The authors show that the PHD1 of AIRE has E3 ligase activity, which is abolished by common disease-causing mutations. By contrast, PHD2 is responsible for transcriptional activation. It remains to be determined whether the E3 ligase activity modifies proteins in the AIRE-containing transcriptional complex or has an independent effect.

\section{TUMOUR IMMUNITY}

Immune recognition of a human renal cancer antigen through post-translational protein splicing.

Hanada, K. et al. Nature 427, 252-256 (2004)

This study indicates a new mechanism by which protein epitopes can be generated for presentation on MHC class I molecules. A human cytotoxic T lymphocyte (CTL) clone isolated from a renal cell carcinoma that overexpresses fibroblast growth factor 5 (FGF5) was tested for recognition of HLA-A3-expressing cells transfected with various truncated and internally deleted forms of FGF5. Surprisingly, the CTLs were shown to recognize an FGF5 peptide consisting of five amino-terminal amino acids and four carboxy-terminal amino acids. Further experiments showed that this epitope was created by post-translational protein splicing the first demonstration of such a process occurring in vertebrates. The ability of the immune system to recognize non-contiguous peptide sequences could potentially enhance immune surveillance.

\section{T-CELL RESPONSES}

\section{GATA-3 deficiency abrogates the development and} maintenance of Thelper type 2 cells.

Pai, S.-Y. et al. Proc. Natl Acad. Sci. USA 101, 1993-1998 (2004)

These authors have used the conditional knockout of Gata3 in mice to confirm the role of this transcription factor in the commitment of naive $\mathrm{CD} 4{ }^{+} \mathrm{T}$ cells to the Thelper $2\left(\mathrm{~T}_{\mathrm{H}} 2\right)$ lineage. In previous studies, overexpression of Gata3 in developing $\mathrm{T}_{\mathrm{H}} 1$ cells was sufficient to drive $\mathrm{T}_{\mathrm{H}} 2$-type cytokine expression. However, confirmation of the physiological role of Gata3 has been lacking, as deletion of Gata3 results in an early block in T-lineage commitment. Conditional deletion of Gata 3 in stage- 3 doublenegative thymocytes led to both $\mathrm{CD} 4^{+}$and $\mathrm{CD} 8^{+} \mathrm{T}$ cells that were unable to produce type- 2 cytokines. Furthermore, the persistent expression of Gata3 was required for $\mathrm{T}_{\mathrm{H}} 2$ cells to maintain their appropriate cytokine production, indicating that Gata3 is required for both the development and active maintenance of $\mathrm{T}_{\mathrm{H}} 2$ cells. 